\title{
PREVALÊNCIA DE FLEBITE RELACIONADA AO USO DE DISPOSITIVOS INTRAVENOSOS PERIFÉRICOS EM CRIANÇAS
}

\author{
Elessandra Souza Bitencourt ${ }^{1}$, Camila Nunes Leal ${ }^{1}$, Radamés Boostel ${ }^{2}$, Verônica de Azevedo Mazza ${ }^{3}$, Jorge \\ Vinícius Cestari Felix ${ }^{4}$, Edivane Pedrolo ${ }^{5}$
}

\begin{abstract}
RESUMO: Objetivo: Avaliar a prevalência de flebite relacionada ao uso de dispositivos intravenosos periféricos em crianças em um hospital universitário. Método: Estudo descritivo e retrospectivo, quantitativo, com base em dados provenientes da ficha de notificação de flebite relacionada à terapia intravenosa periférica, no período de junho de 2011 a junho de 2014, da unidade de terapia intensiva pediátrica, na cidade de Curitiba-PR. Resultados: De um total de 1306 cateteres, foram notificados 339 casos de flebite (prevalência de 26\%) no período de três anos. A prevalência de flebite foi de $34 \%$ nas crianças de 0 a 2 anos e de $30,2 \%$ ( $n=179$ ) no sexo feminino. Quanto à classificação das flebites $82,6 \%(n=280)$ eram de Grau 1 e o tempo médio de permanência do dispositivo foi de 49,92 \pm 43,19 horas. Conclusão: Os dados apresentados demonstram alta prevalência de flebite em pacientes pediátricos e a necessidade de buscar medidas que reduzam esses eventos.
\end{abstract}

DESCRITORES: Segurança do paciente; Flebite; Enfermagem pediátrica; Cateterismo venoso periférico.

\section{PREVALENCE OF PHLEBITIS RELATED TO THE USE OF PERIPHERAL INTRAVENOUS DEVICES IN CHILDREN}

ABSTRACT: Objective: To assess the prevalence of phlebitis, related to the use of peripheral intravenous devices in children in a teaching hospital. Method: A quantitative, descriptive and retrospective study, based in data taken from the phlebitis notification form related to peripheral intravenous therapy, in June 2011 - June 2014, in a pediatric intensive care unit in the city of Curitiba, in the Brazilian state of Paraná (PR). Results: Of a total of 1306 catheters, 339 cases of phlebitis were notified (prevalence of $26 \%$ ) in the threeyear period. The prevalence of phlebitis was $34 \%$ in children aged from 0 to 2 years and was $30.2 \%(n=179)$ among female children. Regarding the classification of the cases of phlebitis, $82.6 \%(n=280)$ were Grade 1 , and the mean dwell-time of the device was $49.92 \pm$ 43.19 hours. Conclusion: The data presented here demonstrate a high prevalence of phlebitis in pediatric patients, and the need to seek measures capable of reducing these events.

DESCRIPTORS: Patient safety; Phlebitis; Pediatric nursing; Catheterization, peripheral.

\section{PREVALENCIA DE FLEBITIS RELACIONADA AL USO DE DISPOSITIVOS INTRAVENOSOS PERIFÉRICOS EN NIÑOS}

RESUMEN: Objetivo: Evaluar la prevalencia de flebitis relacionada al uso de dispositivos intravenosos periféricos en niños, en un hospital universitario. Métodos: Estudio descriptivo y retrospectivo, cuantitativo, basado en datos obtenidos de ficha de notificación de flebitis relacionada a terapia intravenosa periférica, desde junio de 2011 a junio de 2014, en unidad de terapia intensiva pediátrica, ciudad de Curitiba-PR. Resultados: Sobre un total de 1306 catéteres, fueron notificados 339 casos de flebitis (prevalencia del $26 \%$ ) en un lapso de tres años. La prevalencia de flebitis fue del $34 \%$ en niños de 0 a 2 años y de 30,2\% ( $n=179$ ) en el sexo femenino. Respecto a la clasificación de las flebitis, $82,6 \%(n=280)$ fueron de Grado 1, y el tiempo medio de permanencia del dispositivo fue de $49,92 \pm$ 43,19 horas. Conclusión: Los datos presentados demuestran alta prevalencia de flebitis en pacientes pediátricos, y la necesidad de buscar medidas reductoras de tales eventos.

DESCRIPTORES: Seguridad del Paciente; Flebitis; Enfermería Pediátrica; Cateterismo Venoso Periférico.

1'Discente de Enfermagem. Universidade Federal do Paraná. Curitiba, PR, Brasil.

${ }^{2}$ Enfermeiro. Mestrando em Enfermagem. Universidade Federal do Paraná. Curitiba, PR, Brasil.

${ }^{3}$ Enfermeira. Doutora em Enfermagem. Docente de Enfermagem e do Programa de Pós Graduação em Enfermagem da Universidade Federal do Paraná. Curitiba, PR, Brasil.

${ }^{4}$ Enfermeiro. Doutor em Ciências - Fisiologia Humana. Docente de Enfermagem e do Programa de Pós Graduação em Enfermagem da Universidade Federal do Paraná. Curitiba, PR, Brasil.

${ }^{5}$ Enfermeira. Doutoranda em Enfermagem. Universidade Federal do Paraná. Curitiba, PR, Brasil.

Autor Correspondente:

Recebido: $29 / 11 / 2016$

Radamés Boostel

Universidade Federal do Paraná

Finalizado: 09/11/2017

Av. Prefeito Lothário Meissner, 632 - 80.210-170 - Curitiba, PR, Brasil

E-mail: radames.boostel@gmail.com 


\section{INTRODUÇÃO}

O uso de dispositivos intravenosos periféricos (DIVP) em pediatria é uma prática frequente em ambiente hospitalar, sendo necessário para a administração de drogas e fluidos pela via intravenosa ${ }^{(1)}$. Complicações decorrentes da terapia intravenosa (TIV) são resultados não desejados, porém comuns, relacionados a diversos fatores de risco tais como a natureza dos fármacos, a duração da terapia, as características individuais de cada paciente, a habilidade técnica do profissional, o local da punção, o tipo e o calibre do DIVP utilizado, a manipulação e fixação do acesso venoso, entre outros ${ }^{(2-4)}$.

Dentre as complicações da TIV tem-se a flebite, que apesar de não se caracterizar como a complicação mais frequente na população pediátrica ${ }^{(5)}$ acarreta importantes consequências para os pacientes. Dados sobre flebite nesta população são incipientes, no entanto há relatos de prevalência deste agravo na população brasileira de $2,7 \% \%^{(5)}, 3,8 \%{ }^{(6)}$ e até $63 \%$ dos pacientes em uso de DIVP(7). Seus principais sinais e sintomas são dor, eritema, calor, edema, endurecimento, aparecimento de cordão palpável, exsudato purulento e velocidade de infusão lenta ${ }^{(8)}$. A flebite é classificada em graus de 0 até 4, conforme a gravidade ${ }^{(9)}$, e pode ser de etiologia mecânica, química ou infecciosa ${ }^{(8)}$.

A TIV em pacientes pediátricos se constitui um desafio devido às características específicas desta população. Fatores como a maior fragilidade capilar, a rede venosa de pequeno calibre e de difícil visualização, a adiposidade aumentada, pouca cooperação da criança e o estresse parental aumentam a complexidade da situação, desde o procedimento de punção venosa, que se torna mais difícil e demorado, até a fixação, utilização e manutenção adequada do DIVP(1). Além disso, observa-se maior incidência de complicações relacionadas à TIV em crianças mais novas, menores de um ano, quando comparadas a crianças de mais de cinco anos ${ }^{(10)}$.

Nesse sentido, reduzir a incidência de complicações e a ocorrência de múltiplas/novas punções é um grande desafio para a equipe de enfermagem e uma necessidade premente na busca da melhoria da qualidade da assistência e da segurança do paciente. Tendo em vista as altas taxas de insucesso/ complicações da TIV relatadas na literatura, este estudo tem por objetivo avaliar a prevalência de flebite relacionada ao uso de DIVP em crianças de um hospital universitário da cidade de Curitiba-PR.

\section{- MÉTODO}

Trata-se de um estudo descritivo retrospectivo, de abordagem quantitativa. A fonte de dados secundários foram as fichas de notificação de flebite relacionada à terapia intravenosa periférica de uma unidade de terapia intensiva (UTI) pediátrica de um hospital universitário do Sul do Brasil. O período de coleta de dados foi de julho a dezembro de 2014.

Incluíram-se todas as fichas de notificação de flebite da UTI pediátrica, desde junho de 2011, início de sua implantação, até junho de 2014. Nesta unidade admitem-se crianças com idades que variam de 29 dias até 14 anos. Não houve critérios de exclusão.

Foram analisados e tabulados todos os dados da ficha de notificação de flebite, a qual contém informações referentes ao paciente e ao evento adverso/incidente com a TIV ou com o DIVP, constando a data da ocorrência e da punção venosa, os medicamentos em curso, o tipo de dispositivo utilizado, fatores de risco individuais envolvidos e uma tabela de classificação da flebite. Os prontuários dos pacientes foram consultados para informações adicionais e caracterização da amostra com relação ao seu perfil sociodemográfico e clínico.

A avaliação do sítio de inserção foi realizada pelos enfermeiros da unidade, e utilizou-se a Escala de Maddox para classificação do grau de flebite, conforme segue: Grau 0 - Sem sinais e sintomas; Grau 1 Dor no local, eritema ou edema, sem endurecimento e cordão fibroso não palpável; Grau 2 - Dor local, eritema ou edema, formação de endurecimento e cordão fibroso não palpável; Grau 3 - Dor local, eritema ou edema, formação de endurecimento e cordão fibroso palpável ${ }^{(11)}$.

A prevalência de flebite foi calculada pela fórmula: (número de casos de flebite/ número de pacientesdia com acesso periférico) x $100^{(12)}$. Os dados foram tabulados e expressos na forma de gráficos e tabelas, 
com auxílio dos programas Microsoft Exce ${ }^{\circledR}$ e GraphPad Prism $4^{\circledR}$, sendo posteriormente analisados mediante estatística descritiva e apresentados na forma de valores absolutos e porcentagens.

A pesquisa cumpriu os preceitos éticos, foi aprovada em 11 de junho de 2014 pelo Comitê de Ética em Pesquisa do Setor de Ciências da Saúde sob o parecer n 722356/2014.

\section{RESULTADOS}

No período de junho de 2011 a junho de 2014, houve 871 internamentos na UTI pediátrica, segundo dados fornecidos pelo Serviço de Estratégia e Planejamento (SEPLAN) do hospital onde foi realizada a pesquisa. Foram utilizados 1306 cateteres periféricos flexíveis, o que corresponde a uma média de 1,5 cateteres por paciente por internação.

Neste mesmo período, 339 casos de flebite foram registrados nas fichas de notificação na UTI pediátrica. Destes, $52,8 \%(n=179)$ eram do sexo feminino. A idade variou entre o mínimo de 29 dias e o máximo de 14 anos. 52,5\% ( $n=178)$ apresentou idade de 0 a 2 anos, seguida de $19,5 \%(n=66)$ com idade entre 5 e 10 anos. Com relação aos internamentos, as principais causas foram as doenças respiratórias $(36,3 \%, \mathrm{n}=123)$, as doenças hematológicas $(9,5 \%, \mathrm{n}=32)$ e os distúrbios metabólicos $(9,2 \%, \mathrm{n}=31)$ (Tabela 1). O tempo médio de permanência na unidade foi de 11 dias e variou entre o mínimo de 1 e máximo de 59 dias.

Tabela 1 - Caracterização da amostra segundo o perfil clínico e sociodemográfico. Curitiba, PR, Brasil, 2011-2014

\begin{tabular}{lcc} 
Variável & $\mathbf{n}$ & $\%$ \\
\hline Sexo & & \\
\hline Masculino & 160 & 47,2 \\
\hline Feminino & 179 & 52,8 \\
\hline Total & 339 & 100 \\
\hline Idade & & \\
\hline 0 a 2 anos & 178 & 52,5 \\
\hline 2 a 5 anos & 47 & 13,9 \\
\hline 5 a 10 anos & 66 & 19,5 \\
\hline$>10$ anos & 48 & 14,1 \\
\hline Total & 339 & 100 \\
\hline Motivo da Internação & & \\
\hline Doenças Respiratórias & 123 & 36,3 \\
\hline Doenças Cardiovasculares & 9 & 2,7 \\
\hline Doenças Hematológicas & 32 & 9,5 \\
\hline Distúrbios Metabólicos & 31 & 9,2 \\
\hline Neoplasias & 26 & 7,7 \\
\hline Cirurgias & 27 & 7,9 \\
\hline Doenças neurológicas & 22 & 6,5 \\
\hline Doenças gastrointestinais & 13 & 3,8 \\
\hline Choque séptico & 10 & 2,9 \\
\hline Doenças infectocontagiosas & 18 & 5,3 \\
\hline Síndromes raras & 28 & 8,2 \\
\hline Total & 339 & 100 \\
\hline & &
\end{tabular}

A prevalência de flebite no período foi de $26 \%$ (339 notificações em 1306 cateteres). Observou-se prevalência em crianças do sexo feminino $(30,2 \%, n=179)$. Com relação à idade, verificou-se prevalência entre 0 e 2 anos $(34,5 \%, n=178)$ (Tabela 2$)$. 
Tabela 2 - Prevalência de flebite com relação ao sexo e faixa etária. Curitiba, PR, Brasil, 2011-2014

\begin{tabular}{|c|c|c|c|c|c|c|}
\hline \multirow[b]{2}{*}{ Sexo } & \multicolumn{2}{|c|}{ Pacientes } & \multicolumn{2}{|c|}{ Cateteres utilizados } & \multirow{2}{*}{$\begin{array}{c}\begin{array}{c}\text { Casos de } \\
\text { Flebite }\end{array} \\
\mathrm{N}\end{array}$} & \multirow{2}{*}{$\begin{array}{c}\text { Prevalência } \\
\%\end{array}$} \\
\hline & $\mathrm{n}$ & $\%$ & $\mathrm{n}$ & $\%$ & & \\
\hline Masculino & 476 & 56,7 & 714 & 54,7 & 160 & 22,4 \\
\hline Feminino & 395 & 45,3 & 592 & 45,3 & 179 & 30,2 \\
\hline Total & 871 & 100 & 1306 & 100 & 339 & - \\
\hline Prevalência geral & - & - & - & - & - & 26 \\
\hline \multicolumn{7}{|l|}{ Idade } \\
\hline $0 \geq 2$ anos & 344 & 39,5 & 516 & 39,5 & 178 & 34,5 \\
\hline $2 \geq 5$ anos & 125 & 14,4 & 187 & 14,3 & 47 & 25,13 \\
\hline $5 \geq 10$ anos & 174 & 20 & 261 & 20 & 66 & 25,3 \\
\hline$>10$ anos & 228 & 26,2 & 342 & 26,2 & 48 & 14 \\
\hline Total & 871 & 100 & 1306 & 100 & 339 & - \\
\hline
\end{tabular}

Considerados os casos de notificação de flebite com relação à sua classificação, o maior número de ocorrências $(82,6 \%, \mathrm{n}=280)$ foi o de flebite Grau 1 (Figura 1 ).

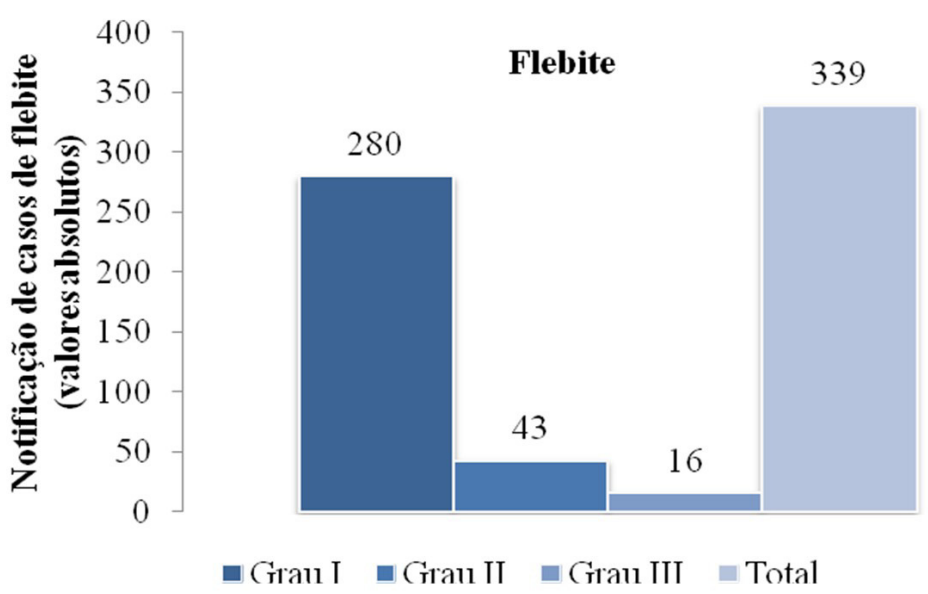

Figura 1 - Classificação dos casos notificados de flebite. Curitiba, PR, Brasil, 2011-2014

O tipo de terapia mais frequente foi a contínua, correspondendo a $83,1 \%(n=282)$ dos dispositivos utilizados. O tempo de permanência dos dispositivos intravenosos periféricos foi avaliado em horas, e a média foi de 49,92 $\pm 43,19$ horas, com mínimo de 1 hora e máximo de 278 horas. A maior parte dos cateteres $(34,3 \%, \mathrm{n}=116)$ permaneceu na faixa entre 24 e 48 horas. Um número expressivo de dispositivos $(23,3 \%, \mathrm{n}=79)$ foi retirado em menos de 24 horas (Tabela 3$)$.

Tabela 3 - Variáveis relacionadas ao dispositivo intravenoso periférico. Curitiba, PR, Brasil, 2011-2014 (continua)

\begin{tabular}{lcccccc} 
Variável & \multicolumn{5}{c}{ Tipo de dispositivo intravenoso periférico } \\
\hline & Flexível (poliuretano) & Rígido (agulhado) & Total & $\%$ \\
\hline Tipo de Terapia & $\mathrm{n}$ & $\%$ & $\mathrm{n}$ & $\%$ & $\mathrm{n}$ & \\
\hline Contínua & 280 & 82,5 & 2 & 0,6 & & \\
\hline Intermitente & 55 & 16,3 & 2 & 0,6 & 339 \\
\hline Total & 335 & 98,8 & 4 & 1,2 & 100 \\
\hline \hline
\end{tabular}




\begin{tabular}{|c|c|c|c|c|c|c|}
\hline \multicolumn{7}{|c|}{ Tempo de Permanência do Dispositivo Intravenoso Periférico } \\
\hline$<24$ horas & 77 & 22,7 & 2 & 0,6 & & \\
\hline 24 a 48 horas & 115 & 34 & 1 & 0,3 & & \\
\hline 48 a 72 horas & 46 & 13,5 & - & - & & \\
\hline 72 a 96 horas & 25 & 7,4 & - & - & & \\
\hline$>96$ horas & 28 & 8,2 & - & - & & \\
\hline Não identificado & 44 & 13 & 1 & 0,3 & & \\
\hline Total & 335 & 98,8 & 4 & 1,2 & 339 & 100 \\
\hline
\end{tabular}

Com relação às drogas ou soluções terapêuticas administradas, as mais frequentes foram as soluções eletrolíticas $(51,3 \%, n=174)$, seguidas da associação de duas ou mais drogas $(13 \%, n=44)$, incluindo sedativos, drogas vasoativas e antimicrobianos ou cada uma delas isoladamente (Tabela 4).

Tabela 4 - Soluções terapêuticas encontradas nas notificações de flebite. Curitiba, PR, Brasil, 2011-2014

\begin{tabular}{lcc} 
Solução terapêutica ou medicamento & $\mathbf{n}$ & $\mathbf{\%}$ \\
\hline Eletrólitos & 174 & 51,3 \\
\hline Sedativos & 21 & 6,2 \\
\hline Drogas vasoativas & 16 & 4,7 \\
\hline Antimicrobianos & 15 & 4,5 \\
\hline Corticoides & 7 & 2 \\
\hline Broncodilatadores & 7 & 2 \\
\hline Nutrição Parenteral Total & 6 & 1,8 \\
\hline Hemoderivados & 3 & 0,9 \\
\hline Anticonvulsivantes & 3 & 0,9 \\
\hline Analgésicos & 2 & 0,6 \\
\hline Associação de duas ou mais drogas & 44 & 13 \\
\hline Outras drogas & 6 & 1,8 \\
\hline Não identificado & 35 & 10,3 \\
\hline Total & 339 & 100
\end{tabular}

\section{DISCUSSÃO}

A Infusion Nurses Society (INS) estabelece os padrões internacionais para a documentação da terapia intravenosa, que devem incluir não apenas os sinais e sintomas clássicos de flebite, mas também informações relacionadas à população da qual o paciente faz parte. Deve-se considerar os fatores de risco, o tipo de terapia e de drogas utilizadas, o método de administração/infusão, o preparo do local de punção, o tipo de dispositivo, a prevenção de complicações, as intervenções realizadas, a resposta do paciente ao tratamento, dentre outros ${ }^{(9)}$.

Ao utilizarmos a ficha de notificação de flebite da UTI pediátrica para a coleta de dados, observamos que faltam informações mais detalhadas sobre as condições do paciente, tais como o calibre do dispositivo utilizado, o local da punção e o tipo de fixação do acesso venoso.

A Agência Nacional de Vigilância Sanitária (ANVISA) recomenda que as instituições de saúde realizem o monitoramento de eventos adversos ocorridos com os pacientes ${ }^{(13)}$. O enfermeiro possui papel fundamental no processo de gerenciamento e manutenção da terapia intravenosa, o qual se realizado e registrado de forma adequada terá impacto direto na redução dos casos de flebite ${ }^{(14)}$. Para 
tanto, é necessário que haja a organização de registros completos em documentação específica, sobre todos os aspectos da TIV, permitindo a identificação de todos os possíveis fatores que levaram ao incidente, conforme preconizado pela Resolução $\mathrm{n}^{\circ} 514$ do Conselho Federal de Enfermagem ${ }^{(15)}$.

Estudo desenvolvido em um hospital pediátrico privado, no período antes e após um processo de acreditação hospitalar, demonstrou redução da incidência de flebite, considerada um indicador da qualidade do cuidado prestado, de aproximadamente 23 casos a cada 100 pacientes com cateter para próximo de zero, com eventos esporádicos em um período de cinco anos. Os autores destacam que esta redução pode ser devida a implantação de protocolos institucionais e guidelines de cuidado na instituição durante o processo de acreditação ${ }^{(16)}$. Assim, demonstra-se a importância da instituição de rotinas de cuidado como ferramenta para prevenção de flebite.

Os resultados desta pesquisa demonstram uma prevalência de flebite de $26 \%$ em crianças. A literatura difere muito neste aspecto, sendo que alguns trabalhos reportam taxas de flebite de $1,5 \%{ }^{(4)}$, $2,7 \%{ }^{(5)}, 3,8 \%{ }^{(6)}$, entre $8,4 \%$ e $40,5 \%{ }^{(17)}, 63 \%{ }^{(7,18)}$ e até $71 \%^{(19)}$. Destaca-se que esta grande divergência entre os estudos se deve aos critérios de diagnósticos empregados e a realidade de cada instituição estudada. A INS preconiza que as taxas de prevalência de flebite não devem ultrapassar os $5 \%$, considerando-se qualquer população ${ }^{(9)}$. Nesse sentido, aprimorar os processos relacionados à TIV em todos os seus aspectos visando à redução das complicações e a melhoria das taxas de sucesso continua sendo um desafio para as instituições de saúde, e em especial para a Enfermagem.

Ao analisar a prevalência de flebite de acordo com a idade, evidenciou-se maior número de casos desta complicação em crianças com idade até dois anos, o que corrobora com a literatura em que complicações por DIVP são mais observadas em pacientes mais jovens ${ }^{(10)}$. No entanto, estudo com 80 crianças não demonstrou relação entre a idade e o grau de flebite apresentado ${ }^{(18)}$. Quanto ao gênero, o sexo feminino apresentou maior prevalência de flebite, sendo este dado também encontrado em estudo que avaliou fatores predisponentes para flebite associada aos DIVP(5).

Com relação ao tempo de permanência dos DIVPs, tanto o Center for Disease Control and Prevention (CDC) quanto a Agência Nacional de Vigilância Sanitária (ANVISA) recomendam que a troca de cateteres periféricos em pacientes pediátricos deve ser realizada apenas com indicação clínica e não rotineiramente de 72 a 96 horas $^{(2,20)}$. Uma revisão sistemática atualizada, com base em ensaios clínicos randomizados em adultos, também demonstrou não haver diferença significativa nas taxas de infecção da corrente sanguínea ou de flebite relacionadas ao uso de cateteres venosos periféricos quando a troca é realizada de rotina, entre 72-96 horas, ou quando realizada apenas com indicação clínica ${ }^{(21)}$.

Estudo recente realizado em crianças demonstrou taxas de insucesso dos DIVPs de aproximadamente $25 \%$ devido às complicações, sendo que o tempo médio de permanência dos dispositivos foi de 29 horas $^{(4)}$. Este mesmo estudo, em contraposição aos estudos anteriores, apontou a flebite como a quarta causa de retirada do cateter, sendo superada pela infiltração em primeiro lugar, seguida da retirada (deslocamento) acidental, e da oclusão ${ }^{(4)}$. Nesta pesquisa o tempo médio de permanência do cateter foi de 49,92 $\pm 43,19$ horas, o que é superior aos dados encontrados no estudo supracitado e, embora esse dado denote maior viabilidade/sucesso dos DIVPs, pode ter sido um fator contribuinte para a maior prevalência de flebite nesta pesquisa. A maior parte dos dispositivos permaneceu por um tempo entre 24 a 48 horas, mas uma quantidade (22\%) foi retirada com tempo inferior a 24 horas.

Um dado relevante é o uso de fármacos que possuem $\mathrm{pH}$ e osmolaridade muito diferentes do sangue, levando a um risco aumentado para flebite ${ }^{(22)}$. Nesta pesquisa, entre as drogas que estavam em curso no momento da notificação, destaca-se o uso de eletrólitos, a associação de um ou mais medicamentos, sedativos, drogas vasoativas e antimicrobianos. Corroborando com nossos achados, outros estudos encontraram associação entre a infusão de fenitoína, manitol ${ }^{(20)}$, antibióticos ${ }^{(7,23-24)}$, cloreto de potássio ${ }^{(19,24)}$ e soluções hipertônicas ${ }^{(24)}$ com casos de flebites.

Conhecer o pH das drogas, a quantidade e o diluente adequado são importantes para minimizar os riscos de flebite, visto que quanto mais ácido, faz-se necessária a diluição do medicamento em maior volume possível tolerado clinicamente pelo paciente ${ }^{(7,25)}$. Diversos estudos na literatura destacam que agentes antimicrobianos possuem $\mathrm{pH}$ ácido e são responsáveis por grande parte dos processos de flebite de etiologia química em crianças $^{(5,7,22)}$.

Estudo de revisão sistemática evidenciou que os casos de flebite em crianças estão relacionados 
à diluição e infusão de drogas de forma inadequada, bem como a administração de mais de um medicamento com potencial irritativo para veia pelo mesmo acesso venoso, fatos que contribuem para ocorrência desta complicação. Assim, destaca a necessidade de instituir protocolos de diluição e reconstituição de drogas, com vistas a atuar de forma preventiva ${ }^{(26)}$. Esta realidade corrobora com os dados encontrados na presente pesquisa e pode estar relacionada à elevada prevalência de flebite na população estudada.

Nesta pesquisa encontrou-se uma prevalência de flebite de $26 \%$, dado acima do recomendado pela literatura, no entanto evidenciou-se que $82,6 \%$ das flebites notificadas foram de Grau 1, não acarretando maior gravidade dos casos. Estudo prospectivo desenvolvido com 80 crianças identificou o Grau 1 de flebite como o mais frequente, com 46,25\% ( $n=37)$, entretanto apresentou índices elevados de flebite Grau $2(23,75 \%)^{(19)}$. Ressalta-se a importância da vigilância constante e observação do DIVP, com vistas a identificar os sinais e sintomas de flebite de forma precoce, e assim minimizar os danos ao paciente.

Como limitação para o estudo, observou-se que o instrumento de notificação de flebite utilizado não continha dados como tamanho do dispositivo, cobertura aplicada, local da punção e profissional responsável pelo procedimento, o que impossibilitou uma melhor avaliação dos eventos adversos. No mais, os diferentes critérios diagnósticos empregados nos estudos dificultam a comparação e discussão dos resultados encontrados.

\section{CONCLUSÃO}

A prevalência de flebite na presente pesquisa foi elevada $(26 \%)$, com prevalência em crianças do sexo feminino e em menores de dois anos. Portanto, buscar medidas que reduzam esses eventos é fundamental para garantir um atendimento de qualidade, diminuir a necessidade de múltiplas punções, a dor dos pacientes e familiares, o tempo de internamento, os custos hospitalares, dentre outras variáveis.

Os dados apresentados demonstram a necessidade de melhorias no instrumento de notificação dos casos de flebite, assim como na prática profissional, no intuito de buscar atingir as metas recomendadas pela INS. Neste sentido, conhecer as tecnologias que são utilizadas, os fármacos e suas interações com outras medicações, tempo e volume de infusão recomendada, adotar técnicas assépticas, escolher de forma adequada o calibre do cateter e o sítio de punção, e utilizar as melhores evidências científicas podem colaborar com o sucesso na diminuição de flebites relacionadas ao uso de DIVP. A implantação de protocolos institucionais e guidelines de cuidado que visem à prevenção da flebite são essenciais para um cuidado seguro.

Considerando que a flebite é um evento adverso possível de prevenção e que a enfermagem tem papel central no cuidado com a terapia intravenosa, esta pesquisa contribui para uma reflexão e busca por melhorias no cuidado à criança em uso dos DIVPs.

\section{REFERÊNCIAS}

1. Reigart JR, Chamberlain KH, Eldridge D, O'Brien ES, Freeland KD, Larsen $P$, et al. Peripheral intravenous access in pediatric inpatients. Clin. Pediatr (Phila). [Internet] 2012;51(5) [acesso em 20 mai 2015]. Disponível: http://dx.doi. org/10.1177/0009922811435164.

2. O'Grady NP, Alexander M, Burns LA, Dellinger EP, Garland J, Heard SO, et al. Guidelines for de prevention of intravascular catheter-related infections. Clin Infect Dis. [Internet] 2011;52(9) [acesso em 20 mai 2015]. Disponível: http://dx.doi.org/10.1093/cid/cir257.

3. Modes PSSA, Gaiva MAM, Rosa MKO, Granjeiro CF. Cuidados de enfermagem nas complicações da punção venosa periférica em recém-nascidos. Rev Rene. [Internet] 2011;12(2) [acesso em 15 mai 2015]. Disponível: http:// www.revistarene.ufc.br/vol12n2_html_site/a14v12n2.htm.

4. Malyon L, Ullman AJ, Phillips N, Young J, Kleidon T, Murfield J, et al. Peripheral intravenous catheter duration and failure in paediatric acute care: a prospective cohort study. Emerg Med Australas. [Internet] 2014;26(6) [acesso 
em 28 abr 2015]. Disponível: http://dx.doi.org/10.1111/1742-6723.12305.

5. Jacinto AKL, Avelar AFM, Wilson AMMM, Pedreira MLG. Phlebitis associated with peripheral intravenous catheters in children: study of predisposing factors. Esc. Anna Nery. [Internet] 2014;18(2) [acesso em 13 jun 2015$].$ Disponível: http://dx.doi.org/10.5935/1414-8145.20140032.

6. de Almeida TJC, Miranda JOF, dos Santos LM, de Santana RCB, de Camargo CL, Nascimento Sobrinho CL. Peripheral venous accesses in hospitalized children: a photographic study. Rev Enferm UFPE on line. [Internet] 2016;10(Suppl 2) [acesso em 3 nov 2016]. Disponível: http://www.revista.ufpe.br/revistaenfermagem/index.php/ revista/article/viewArticle/8401.

7. Martins TSS, Silvino ZR, Dias LS. Profile of intravenous therapy in a pediatric university hospital and association with the occurrence of infusion failures: quantitative study. Online Braz J Nurs. [Internet] 2010;9(2) [acesso em 13 jun 2015]. Disponível: http://www.objnursing.uff.br/index.php/nursing/article/view/3067/684.

8. dos Reis PE, Silveira RC, Vasques Cl, de Carvalho EC. Pharmacological interventions to treat phlebitis: a systematic review. J Infus Nurs. [Internet] 2009;32(2) [acesso em 10 mai 2015]. Disponível: http://dx.doi.org/10.1097/ NAN.0b013e318198d497.

9. Alexander M. Infusion nursing: standards of practice-infusion. J Infus Nurs. [Internet] 2011;34(1S) [acesso em 10 mai 2015]. Disponível: https://engage.ahima.org/HigherLogic/System/DownloadDocumentFile. ashx? DocumentFileKey=2238ee0a-c2df-4d1a-affa-f69f2ce41856.

10. Tripathi S, Kaushik V, Singh V. Peripheral IVs: factors affecting complications and patency - a randomized controlled trial. J Infus Nurs. [Internet] 2008;31(3) [acesso em 15 mai 2015]. Disponível: http://dx.doi.org/10.1097/01. NAN.0000317704.03415.b9.

11. Maddoxx RR, Rush DR, Rapp RP, Foster TS, Mazella V, McKean HE. Double-blind study to investigate methods to prevent cephalothin-induced phlebitis. Am J Hosp Pharm. 1977;34(1):29-34.

12. Associação Paulista de Medicina (APM). Conselho Regional de Medicina do Estado de São Paulo (CREMESP). Manual de indicadores de enfermagem NAGEH. Compromisso com a qualidade hospitalar. $2^{a}$ ed. São Paulo: APM/CREMESP; 2012.

13. Ministério da Saúde (BR). Agência Nacional de Vigilância Sanitária. Resolução da Diretoria Colegiada - RDC nº 36, de 25 de julho de 2013. Institui ações para a segurança do paciente em serviços de saúde e dá outras providências [Internet]. Diário Oficial da União, 2013 [acesso em 13 set 2015]. Disponível: http://bvsms.saude.gov. br/bvs/saudelegis/anvisa/2013/rdc0036_25_07_2013.html.

14. Oliveira ASS, Parreira PMSD. Intervenções de enfermagem e flebites decorrentes de cateteres venosos periféricos: revisão sistemática da literatura. Rev. Enf. Ref. [Internet] 2010;3(2) [acesso em 19 out 2015]. Disponível: http://www.scielo.mec.pt/scielo.php?script=sci_arttext\&pid=S0874-02832010000400015.

15. Conselho Federal de Enfermagem. Resolução n. 514, de 05 de maio de 2016. Aprova o Guia de Recomendações para os registros de enfermagem no prontuário do paciente, com a finalidade de nortear os profissionais de enfermagem. Rio de Janeiro: COFEN; 2016.

16. Alves DFS, Guirardello EB. Ambiente de trabalho da enfermagem, segurança do paciente e qualidade do cuidado em hospital pediátrico. Rev. Gaúcha Enferm. [Internet] 2016;37(2) [acesso em 5 nov 2016]. Disponível: http://dx.doi.org/10.1590/1983-1447.2016.02.58817.

17. Gómez-Neva E, Bayona JG, Rosselli D. Flebitis asociada con accesos venosos periféricos en niños: revisión sistemática de la literatura. Infectio. [Internet] 2015;19(2) [acesso em 1 nov 2016]. Disponível: http://dx.doi. org/10.1016/j.infect.2014.12.002.

18. Panadero A, Iohom G, Taj J, Mackay N, Shorten G. A dedicated intravenous cannula for postoperative use effect on incidence and severity of phlebitis. Anaesthesia. [Internet] 2002;57(9) [acesso em 15 mai 2015 ]. Disponível: http://dx.doi.org/10.1046/j.1365-2044.2002.02786.x.

19. Nagpal P, Khera GK, Kumar Y. A study assess the clinical pattern of phlebitis among children admitted in selected hospital of Ambala, Haryana. Nurs Midwifery Res J. [Internet] 2015;11(2) [acesso em 4 nov 2016]. Disponível: http://medind.nic.in/nad/t15/i2/nadt15i2p68.pdf. 
20. Ministério da Saúde (BR). Agência Nacional de Vigilância Sanitária (ANVISA). Infecção de corrente sanguínea: orientações para prevenção de infecção primária de corrente sanguínea. Unidade de Investigação e Prevenção das Infecções e dos Efeitos Adversos - UIPEA [Internet] 2010 [acesso em 4 abr 2015]. Disponível: http://adcon. rn.gov.br/ACERVO/Suvisa/doc/DOC000000000034032.PDF.

21. Webster J, Osborne S, Rickard CM, New K. Clinically-indicated replacement versus routine replacement of peripheral venous catheters. Cochrane Database Syst Rev [Internet] 2013;(30) [acesso em 28 abr 2015]. Disponível: http://dx.doi.org/10.1002/14651858.CD007798.pub3.

22. Monteiro C, Crepaldi RMC, Avelar AFM, Peterlini MAS, Pedreira MLG. Hydrogen potential of antibiotic solutions subjected to environmental conditions: a preliminary trial. Rev. esc. enferm USP. [Internet] 2012;46(2) [acesso em 12 jun 2015]. Disponível: http://dx.doi.org/10.1590/S0080-62342012000200007.

23. Shenoy S, Karunakar BP. Factors influencing the peripheral venous catheter survival in critically ill children in a pediatric intensive care unit. Indian J Pediatr. [Internet] 2014;81(12) [acesso em 10 jun 2015]. Disponível: http:// dx.doi.org/10.1007/s12098-014-1430-7.

24. Salgueiro-Olivieira A, Parreira P, Veiga P. Incidence of phlebitis in patients with peripheral intravenous catheters: The influence of some risk factors. Aust J Adv Nurs. [Internet] 2012;30(2) [acesso em 15 mai 2015]. Disponível: http://www.ajan.com.au/Vol30/Issue2/4Salgueiro-Oliveira.pdf.

25. da Silva LD, Camerini FG. Analisys the intravenous medication administration in sentinel network hospital. Texto Contexto Enferm. [Internet] 2012;21(3) [acesso em 12 jun 2015]. Disponível: http://dx.doi.org/10.1590/S010407072012000300019.

26. da Silva LS, Barbato MHS, Valente GSC. Nurses' production regarding peripheral venous catheters used in pediatric procedures: systematic review. Rev enferm UFPE on line. [Internet] 2013;7(4) [acesso em 4 nov 2016]. Disponível: http://www.revista.ufpe.br/revistaenfermagem/index.php/revista/article/view/4359. 